\title{
The Nature of the Hydroxyl Group $\pi$-Electron Interaction. Repulsive Instead of Attractive?
}

\author{
RAYMOND J. ABRAHAM ${ }^{a}$ and JAN M. BAKKE ${ }^{b, *}$ \\ a The Robert Robinson Laboratories, The University of Liverpool, Liverpool, England and \\ ${ }^{b}$ Norwegian Institute of Technology, University of Trondheim, N-7034 Trondheim-NTH, Norway
}

The ${ }^{1} \mathrm{H}$ NMR spectrum of 9-fluorenol at high dilution indicates that the molecule exists in one conformer only, with the $\mathrm{OH}$ proton pointing in between the two aryl rings. The IR spectrum shows no intramolecular hydrogen bond. The interaction is proposed to be due to the repulsion of the hydroxyl oxygen lone-pair electrons and the $\pi$-electrons of the aromatic rings. Earlier data on benzyl alcohols and open chain olefinic alcohols are reinterpreted on the basis of this model and it is not necessary to invoke intramolecular hydrogen bonding except in the case of 3-buten-1-ol.

Alcohols containing $\pi$-electron systems $e$.g. benzyl alcohol and allyl alcohol have been proposed to have weak, intramolecular hydrogen bonds between the $\mathrm{OH}$ proton and the $\pi$-electrons. ${ }^{1-3}$ The proposals have mainly been based on IR spectroscopy in the $\mathrm{OH}$ stretching region and also on microwave spectroscopy ${ }^{4,5}$ and electron diffraction. ${ }^{6}$

During our NMR study of benzyl alcohol and 1,2-diarylethanols we found no evidence of intramolecular hydrogen bonding to the $\pi$-electron system, despite the existence of $\mathrm{OH}$ bands in the IR spectra indicative of such bonds. ${ }^{7,8}$ This has led us to reconsider the criteria of weak intramolecular hydrogen bonds in such systems. In the following, we will show that some of the earlier results are not consistent with the existence of such bonds, and we will also propose a new model for the intramolecular interactions.

\footnotetext{
* Author to whom correspondence should be addressed.
}

During our study of 1,2-diarylethanols, only compounds substituted with hydrogen bond acceptors in the ortho-positions on both rings [e.g. 1,2-di(o-methoxyphenyl)ethanol] were found to have an appreciable proportion of an intramolecular hydrogen bonded conformer. The hydrogen bond was manifested both in IR (a band at $\left.3560 \mathrm{~cm}^{-1}\right)$ and the NMR spectra $(20 \%$ of a conformer with the hydroxyl proton pointing in between the two ortho-groups). ${ }^{8}$ From this it appeared to us that the possibility of an internal hydrogen bond to the $\pi$-electron system would increase if there were two aryl rings to accept the hydrogen bond. The obvious compound to investigate for this effect was 9-fluorenol. Indeed, its proton NMR spectrum at high dilution in $\mathrm{CCl}_{4}$ showed a ${ }^{3} J_{\mathrm{CHOH}}$ of $10.75 \mathrm{~Hz}$, indicating the molecule to be locked in one conformation with the hydroxyl proton pointing in between the two aryl rings (Fig. 1). This was, of course, highly indicative of a strong intramolecular hydrogen bond. We also observed the $\mathrm{CHOH}$ coupling at different temperatures and this gave information on the rotamer composition around the $\mathrm{O}-\mathrm{H}$ bond. From these data, the equilibrium constants and thermodynamic parameters were obtained, $\Delta H^{\circ}=-3.2 \mathrm{kcal} \mathrm{mol}^{-1}, \Delta S^{\circ}=-3.5 \mathrm{cal} \mathrm{mol}^{-1} \mathrm{~K}^{-1}$.

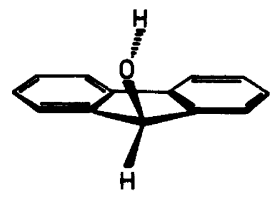

Fig. 1. Predominant conformer (from NMR) of 9-fluorenol. 
Table 1. Hydroxyl stretching absorbtion of alcohols.

\begin{tabular}{llll}
\hline Compound & $v_{\max }\left(\mathrm{cm}^{-1}\right)$ & $\Delta v_{1 / 2}\left(\mathrm{~cm}^{-1}\right)$ & Ref. \\
\hline Methanol & 3643.8 & 22.2 & 12 \\
Ethanol & $3637.3,3627^{a}$ & $18.2,19$ & 12 \\
2-Propanol & $3627.1,3617(\mathrm{sh})$ & $13.8,(2.6)$ & 12 \\
$t$-Butyl alcohol & 3616.9 & 15.2 & 12 \\
Benzyl alcohol & $3636.3(\mathrm{sh}), 3617.1$ & $(24.0), 17.0$ & 12 \\
1,2-Diphenylethanol & 3620 & 26 & 18 \\
1,2-Di $(o-$-nitrophenyl)ethanol & 3615,3540 & $25,49.5$ & 18 \\
1,2-Di-(o-methoxyphenyl)ethanol & 3615,3560 & 25,80 & 18 \\
9-Fluorenol & 3602 & 16 & This work \\
Diphenylmethanol & $3633(\mathrm{sh}), 3615$ & 26 & This work \\
Allyl alcohol & $3634.7(\mathrm{sh}), 3619.4$ & $(23.4), 16.4$ & 15 \\
3-Buten-1-ol & $3635.2,3596.1$ & $24.0,32.3$ & 15 \\
4-Penten-1-ol & $3638.3,3624.5(\mathrm{sh})$ & $20.8,(24.0)$ & 15 \\
5-Hexen-1-ol & $3639.2,3626.0(\mathrm{sh})$ & $20.8,(17.2)$ & 15 \\
\hline
\end{tabular}

${ }^{a}$ Overlapping bands.

These data may have errors due to the uncertainties in $J_{t}$ and $J_{g}$, but they certainly indicate a strong hydrogen bond to be present. However, the chemical shift of the hydroxyl proton $\left(\delta_{\infty}=1.505 \mathrm{ppm}\right)$ was not very different from those of alcohols without such strong bonds (e.g. benzyl alcohol, $1.09 \mathrm{ppm}$, diphenylmethanol, $1.62 \mathrm{ppm}$ ) but well below the ones we have found to have intramolecular hydrogen bonds (e.g. 1,2-di(o-methoxyphenyl)ethanol, $\delta=2.31 \mathrm{ppm}){ }^{8}$ The downfield shift of hydroxyl protons on hydrogen bond formation is well documented. ${ }^{9}$

Further, the IR spectrum at high dilution in $\mathrm{CCl}_{4}$ did not give any indications of such a bond $\left(v_{\max }=3602 \mathrm{~cm}^{-1}, \Delta v_{1 / 2}=16 \mathrm{~cm}^{-1}\right.$, Table 1). Two phenomena are manifested in the IR spectrum of the $\mathrm{OH}$ stretch region if a hydrogen bond is formed: a shift of the band to lower frequency and an increase in the intensity accompanied by an increase in the $\Delta v_{1}$ of the band. The magnitude of these changes is connected to the strength of the hydrogen bond, although no simple relationship exists. ${ }^{10}$ In Table 1 , the $\mathrm{OH}$ stretch frequency together with $\Delta v_{1 / 2}$ are given for a selection of alcohols. From that, it is clear that the frequency and particularly $\Delta v_{1 / 2}$ for 9-fluorenol are not very different from those of alcohols without internal hydrogen bonds, e.g. 1,2diphenylethanol and diphenylmethanol. From its NMR spectrum (see experimental) diphenylmethanol has its hydroxyl proton pointing away from one of the phenyl rings and towards the

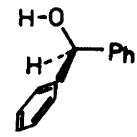

Fig. 2. Predominant rotamer around the $\mathrm{C}-\mathrm{OH}$ bond (from NMR) of diphenylmethanol.

edge of the other without possibility for an internal hydrogen bond (Fig. 2). On the other hand, for 1,2-di(o-methoxyphenyl)ethanol a band at $3560 \mathrm{~cm}^{-1}, \Delta v_{1 / 2}=70 \mathrm{~cm}^{-1}$ was assigned to the internal hydrogen bonded conformer. ${ }^{8}$ These IR data indicate a stronger hydrogen bond than the one in 9-fluorenol. Nevertheless, in 1,2-di( omethoxyphenyl)ethanol only $32 \%$ of the rotamers around the $\mathrm{OH}$ bond participated in a hydrogen bond. ${ }^{8}$ In 9-fluorenol on the other hand, nearly $100 \%$ was in a position where hydrogen bonding was possible. Further, the thermodynamic data for 9-fluorenol corresponds to $\Delta G^{\circ}(298 \mathrm{~K})=-2.2 \mathrm{kcal} \mathrm{mol}^{-1}$. For intermolecular hydrogen bonds $\Delta G^{\circ}(298 \mathrm{~K})=-2.7$ kcal mol-1 corresponded to a shift of $c a .300 \mathrm{~cm}^{-1}$ of the $\mathrm{OH}$ stretching frequency, ${ }^{19}$ much more than the shift observed e.g. in going from diphenylmethanol $\left(3615 \mathrm{~cm}^{-1}\right.$ without an internal hydrogen bond) to 9-fluorenol $\left(3602 \mathrm{~cm}^{-1}\right)$.

If the reason for this conformational homogeneity were indeed an intramolecular hydrogen bond, it must have been a stronger one than in 1,2-di(o-methoxyphenyl)ethanol. It does 
not appear probable that such a bond should not be manifested in the IR and NMR spectra in a more obvious way than is evident in those of 9-fluorenol.

In our opinion, only two explanations of this paradox are possible, either that some hydrogen bonds do not result in the normal changes in the IR and NMR spectra or that the reason for the conformational homogeneity of 9-fluorenol is not an internal hydrogen bond. The first of these would be against all known evidence. ${ }^{1,2,10} \mathrm{We}$ therefore prefer the second explanation and propose the reason to be a repulsion between the $\pi$-electrons of the aryl rings and the lone-pair electrons of the hydroxyl oxygen. These are positioned approximately opposite to the hydroxyl proton. To minimize the repulsion the hydroxyl proton would be placed in between the two aryl rings. The effect of repulsion between the oxygen lone-pair electrons and double bond $\pi$-electrons has been observed for 3-methoxy-1methylenecyclohexanes. ${ }^{11}$ This explanation also applies to other benzyl alcohol systems and to olefinic alcohols. We will treat each of these separately.

Benzyl alcohols. The presence of two $\mathrm{OH}$ bands in the IR spectra of aliphatic alcohols has been convincingly explained as due to several conformers in the solution. ${ }^{12,13}$ In molecules containing double bond systems, such as benzyl alcohols, the low frequency band is $c a .10 \mathrm{~cm}^{-1}$ below the corresponding band in the purely aliphatic alcohols. This has led several authors to propose the existence of weak intramolecular hydrogen bonds, the low frequency band resulting from the bonded conformer. ${ }^{1,2,3}$

Óki has however explained the low frequency band as due to an interaction between the hydroxyl group and the $\pi$-electrons of the aromatic nucleus. The nature of the interaction was not discussed in any detail. ${ }^{14}$ The IR spectra of substituted benzyl alcohols showed two bands, at ca. 3655 and $3615 \mathrm{~cm}^{-1}$. The intensity of these bands was dependent on the substituent. With electron attracting substituents, e.g. p-nitro, the high frequency one was the most intense, but with electron releasing ones e.g. p-methoxy, the opposite was the case. The relationship was successfully correlated with the Hammett $\sigma$ values. ${ }^{14}$ This effect could be ascribed to the existence of a conformer with an internal hydrogen bond. With increasing electron density in the $\pi$-electron system, an increase in the population of the bonded conformer (the one with the low frequency absorption) would take place, as observed. However, if this were the case, the increase in the electron density would lead to a stronger hydrogen bond. This would not only give a higher population of the hydrogen bonded species, but also a shift to lower frequency in the $\mathrm{OH}$ absorption, This was, however, not observed. The bands of $p$-nitrobenzyl alcohol are at the same frequencies as those of $p$-methoxybenzyl alcohol (and of the other ones studied).

We see now that this apparent paradox is resolved if the interaction between the hydroxyl group and the $\pi$-electrons is a repulsion between the lone-pair electrons of the hydroxyl oxygen and the $\pi$-electrons. In p-nitrobenzyl alcohol, with low electron density in the $\pi$-electron system, the repulsion is low and we have an appreciable population of the conformer with the oxygen lone-pair electrons pointing towards the ring and the hydroxyl proton pointing away from it (the so-called free conformer). In $p$-methoxybenzyl alcohol on the other hand, with its relatively high electron density, the repulsion is increased and the conformer with the oxygen lone-pair electrons pointing away from the ring and the hydroxyl proton pointing towards it (the so-called hydrogen bonded conformer) becomes the most populated. One would not expect the frequencies of the $\mathrm{OH}$ bands of the two conformers to be greatly influenced by the electron density of the $\pi$-electron system as the $\mathrm{OH}$ bond is not directly influenced by the repulsion process (as it would have been by the formation of a hydrogen bond). On the other hand the observed correlation of the intensities with the Hammett $\sigma$ values is in accordance with our hypothesis. Our own results from the NMR study of benzyl alcohols also support this, as we found the most abundant conformer to have the hydroxyl proton pointing towards the aromatic ring, but not in that orientation which would be thought to be the best for hydrogen bonding. ${ }^{7}$ The same was the case for 1,2-diarylethanols, the hydroxyl proton pointed towards the 1-aryl ring and the oxygen lone-pair electrons away from it. ${ }^{8}$

Olefinic alcohols. The IR spectra of alcohols of this type have been studied by several authors., ${ }^{3,15}$ Except for 3-buten-1-ol, the open chain ones show absorption above $3600 \mathrm{~cm}^{-1}$ only, and with $\Delta v_{1 / 2}$ from $15-25 \mathrm{~cm}^{-1}$ (Table 1). The spectra 
usually have more than one band in the $\mathrm{OH}$ region, and this has again led to the suggestion of an internal hydrogen bond. Allyl alcohol has also been investigated by microwave ${ }^{4}$ and NMR spectroscopy, ${ }^{16}$ and 3-buten-1-ol by microwave spectroscopy ${ }^{5}$ and electron diffraction. ${ }^{6}$ In both cases, the authors agree that the molecules have a major conformer with the hydroxyl proton pointing towards the double bond. This has again been interpreted as a stabilisation of this conformer by an internal hydrogen bond.

Allyl alcohol has bands at 3634 (sh) and 3619 $\left(\Delta v_{1 / 2}=23 \mathrm{~cm}^{-1}\right)$. From comparison with the other alcohols in Table 1, these bands cannot be taken as indicative of a hydrogen bond strong enough to make the observed conformer the major one. In analogy with the benzyl alcohols, we again propose that the reason for this conformational homogeneity is the repulsion between the lone-pair electrons on the hydroxyl oxygen and the $\pi$-electrons of the double bond.

For 3-buten-1-ol, this cannot be the explanation. Repulsive forces would of course tend to move the lone-pair electrons of the oxygen as far away from the double bond as possible, probably by extending the molecule instead of folding it. The IR spectrum of 3-buten-1-ol is, however, different from that of allyl alcohol (and the other open chain olefinic alcohols) in that it contains a band at significantly lower frequency and with greater bandwidth than observed for the other alcohols, $3596 \mathrm{~cm}^{-1}, \Delta v_{1 / 2}=32 \mathrm{~cm}^{-1} .{ }^{15}$ We interpret this to mean that in 3-buten-1-ol the internal hydrogen bond is strong enough to counteract the repulsion between the lone-pair electrons and the $\pi$-electrons. It is well known that the strength of a hydrogen bond is dependent on the $\mathrm{O}-\mathrm{H}--$ acceptor angle, $180^{\circ}$ being close to the optimal in most cases. ${ }^{17}$ With the $\pi$-electron system as acceptor, the precise point of acceptance is hard to define. It must, however, be more difficult to obtain an angle close to $180^{\circ}$ in systems like allyl alcohol and benzyl alcohol than in the more flexible molecule 3-buten-1-ol. For 4-penten-1-ol and 5-hexen-l-ol, the IR spectra again show only narrow bands at 3638 and $3639 \mathrm{~cm}^{-1}$, respectively. ${ }^{15}$ For these alcohols, the energy loss in the formation of an internal hydrogen bond is larger than that gained and no such bond is formed.

Conclusion. The predominance of one conformer in benzyl alcohols and in allyl alcohol and the
Table 2. Observed ${ }^{3} \mathrm{~J}_{\mathrm{CHOH}}$ and $\delta_{\mathrm{OH}}$ of 9-fluorenol $(5 \mathrm{mM})$ in $\mathrm{CCl}_{4}$ at different temperatures.

\begin{tabular}{rlll}
\hline Temperature $\left({ }^{\circ} \mathrm{C}\right)$ & ${ }^{3} J_{\mathrm{CHOH}}(\mathrm{Hz})$ & $\delta_{\mathrm{OH}}$ & $\Delta v_{1 / 2}(\mathrm{~Hz})$ \\
\hline-20.2 & 11.24 & 1.645 & 1.84 \\
-8.9 & 11.23 & 1.584 & 1.03 \\
2.6 & 10.99 & 1.548 & 0.98 \\
26.0 & 10.75 & 1.505 & 0.78 \\
36.4 & 10.74 & 1.498 & 0.90 \\
48.6 & 10.50 & 1.487 & 0.97 \\
59.2 & 10.50 & 1.477 & 0.98 \\
66.2 & 10.50 & 1.472 & 1.12 \\
\hline
\end{tabular}

predominance of one particular band in the $\mathrm{OH}$ stretching region is not caused by the attractive forces of an intramolecular hydrogen bond. Instead, we propose the reason to be a repulsion between the $\pi$-electrons and the lone-pair electrons of the hydroxyl oxygen.

\section{EXPERIMENTAL}

9-Fluorenol and diphenylmethanol were purchased from Aldrich-Europe and Koch-Light Laboratories, respectively. The NMR and IR spectra were run in $\mathrm{CCl}_{4}$ dried over molecular sieves $\left(3 \mathrm{~A}\right.$, activated at $400{ }^{\circ} \mathrm{C}$ for $4 \mathrm{~h}$ ). A capillary with ${ }^{2} \mathrm{H}_{6}$-benzene supplied the lock signal, and a sphere of molecular sieves (3A) in the sample removed any $\mathrm{OH}$-exchange. The NMR spectra were run on a Bruker $400 \mathrm{MHz}$ or a JEOL FX $100 \mathrm{MHz}$. The IR spectra were run in $\mathrm{CCl}_{4}$ in a $1 \mathrm{~cm}$ Vitreosil cell on a Beckman IR 4250 .

9-Fluorenol $(5 \mathrm{mM})$ had $\delta_{\mathrm{OH}_{\mathrm{x}}}\left(26^{\circ} \mathrm{C}\right)=1.505$ ppm, ${ }^{3} J_{\mathrm{CHOH}}=10.75 \mathrm{~Hz}$, corresponding to $96 \%$ of the conformer with hydroxyl proton trans to the $\mathrm{C}_{9}$ proton $\left({ }^{3} J_{\mathrm{CHOH}-\text { trans }}\right.$ assumed to be 11.24 $\mathrm{Hz}$ and ${ }^{3} J_{\mathrm{CHOH}-\text { gauche }} 2.2 \mathrm{~Hz}^{6}$ ). Diphenylmethanol $(4 \mathrm{mM})$ had $\delta_{\mathrm{OH}_{x}}=1.619 \mathrm{ppm},{ }^{3} \mathrm{~J}_{\mathrm{CHOH}}=3.4$ $\mathrm{Hz}$ corresponding to $14 \%$ of the conformer with the hydroxyl proton trans and $86 \%$ of the one with it gauche to the proton on the hydroxyl bearing carbon. 9-Fluorenol (5 $\mathrm{mM})$ had IR $v_{\max }=3602 \mathrm{~cm}^{-1}, \Delta v_{1 / 2}=16 \mathrm{~cm}^{-1}$, diphenylmethanol ( $4 \mathrm{mM}$ ) had IR $v_{\max }=3633 \mathrm{~cm}^{-1}$ (sh.), 3618 $\mathrm{cm}^{-1}, \Delta v_{1 / 2}=$

$26 \mathrm{~cm}^{-1}$.

The variable temperature experiments were run in $\mathrm{CCl}_{4}$ with a sphere of molecular sieves (3A) and a capillary with ${ }^{2} \mathrm{H}_{4}$-methanol for lock signal. This also served as a temperature monitor as introductory experiments had shown the 
methanol in the capillary to have the same shifts between the $\mathrm{OH}$ and $\mathrm{CH}_{3}$ signals as that observed in a normal methanol sample. The aromatic protons were decoupled during the experiments. The observed ${ }^{3} J_{\mathrm{CHOH}}$ splittings from the $\mathrm{CH}$ and $\mathrm{OH}$ bands were within $0.01 \mathrm{~Hz}$ of each other. The percentage of the two rotamers was calculated from the ${ }^{3} \mathrm{~J}_{\mathrm{CHOH}}$ (see above), $X=$ percentage of trans rotamer. The equilibrium constants, $\mathrm{K}$, were calculated after taking into account that the gauche rotamer has a statistical weight of $2: K=X / \frac{1}{2}(1-X)$. The data from $t=2.6^{\circ} \mathrm{C}$ to $66.2^{\circ} \mathrm{C}$ was used in a van't Hoff plot to obtain $\Delta H^{\circ}=-3.2 \pm 0.4 \mathrm{kcal} \mathrm{mol}^{-1}, \Delta S^{\circ}=$ $-3.5 \pm 1.4 \mathrm{cal} \mathrm{mol}^{-1} \mathrm{~K}^{-1}$, correlation coefficient $r^{2}=0.955$ for the plot.

Acknowledgement. The $400 \mathrm{MHz}$ NMR spectra were obtained at the Norwegian National Laboratory at the University of Trondheim.

\section{REFERENCES}

1. Bellamy, L. J. Advances in Infrared Group Frequencies, Methuen, London 1968.

2. Tichý, M. In Raphael, R. A., Taylor, E. C. and Wynberg, H., Eds., Advances in Organic Chemistry, Methods and Results, Wiley, New York 1965, Vol. 5, p. 115.

3. Joesten, M. D. and Schaad, L. J. Hydrogen Bonding, Decker, New York 1974.

4. Marasimha, Murty, A. and Curl, R. F., Jr. J. Chem. Phys. 46 (1967) 4176.

5. Marstokk, K.-M. and Møllendal, H. Acta Chem. Scand. A 35 (1981) 395.

6. Trætteberg, M. and Østensen, H. Acta Chem. Scand. A 33 (1979) 491.

7. Abraham, R. J. and Bakke, J. M. Tetrahedron 34 (1978) 2947.

8. Abraham, R. J. and Bakke, J. M. Acta Chem. Scand. B 35 (1981) 367.

9. Tucker, E. E. and Lippert, E. In Schuster, P., Zundel, G. and Sandorfy, C., Eds., The Hydrogen Bond, North-Holland, Amsterdam 1976, Vol. 2, Chapter 17 and references therein.

10. Pimentel, G. C. and McClellan, A. L. Annu. Rev. Phys. Chem. 22 (1971) 347.

11. Lambert, J. B. and Taba, K. M. J. Am. Chem. Soc. 103 (1981) 5828.

12. Öki, M. and Iwamura, H. Bull. Chem. Soc. Jpn. 32 (1959) 950.

13. Joris, L., Schleyer, P. v. R. and Ōsawa, E. Tetrahedron 24 (1968) 4759.

14. Ōki, M. and Iwamura, H. Bull Chem. Soc. Jpn. 32 (1959) 955.
15. Oki, M. and Iwamura, H. Bull. Chem. Soc. Jpn. 32 (1959) 567.

16. Pierre, J.-L. Bull. Soc. Chim. Fr. 37 (1970) 3116.

17. Luck, W. A. P. In Schuster, P., Zundel, G. and Sandorfy, C., Eds., The Hydrogen Bond, North-Holland, Amsterdam 1976, Vol. 2, Chapter 11.

18. Bakke, J. M. and Lorentzen, G. B. Acta Chem. Scand. B 28 (1974) 650.

19. Stymne, B., Stymne, H. and Wettermark, G. J. Am. Chem. Soc. 95 (1973) 349.

Received January 1, 1983.

Acta Chem. Scand. B 37 (1983) No. 10 